\title{
TECOPHILAEA CYANOCROCUS LEYB. (TECOPHILACEAE) REDESCUBIERTA EN SU HABITAT NATURAL
}

\author{
TECOPHILAEA CYANOCROCUS LEYB. (TECOPHILACEAE) REDISCOVERED IN \\ ITS NATURAL HABITAT
}

\author{
M. Teresa Eyzaguirre* \& Rosario García de la Huerta
}

RESUMEN

Tecophilaea cyanocrocus Leyb., es una geófita altoandina endémica de la región Metropolitana de Chile. Fue descrita en 1862. Debido al potencial ornamental de sus flores azules, sus cormos fueron extraídos en grandes cantidades y exportados a Europa. Actualmente es cultivada en muchos países. A partir de la mitad del siglo XX se la considera extinguida en la naturaleza. Se da a conocer el descubrimiento de una población de la especie en las montañas de la Región Metropolitana, en la primavera de 2001.

Palabras Claves: Flora de Chile, geófita, especie extinta, Tecophilaea cyanocrocus Leyb.

\section{INTRODUCCION}

Tecophilaea cyanocrocus Leyb., (Figs 1-3), nombre común "Azulillo", corresponde a una planta geófita provista de un cormo con envoltura fibrosa, de 1,2 a $2 \mathrm{~cm}$ de diámetro. Las hojas son 2, lanceoladas, de 7 a $10 \mathrm{~cm}$ de largo y $7 \mathrm{~mm}$ de ancho, en su parte aérea. La base de las hojas y parte del escapo floral están cubiertos por una hoja envainadora, éstos son subterráneos y de un largo de 4 a $12 \mathrm{~cm}$. Las flores se presentan solitarias o en pares, acompañadas de una bráctea similar a las hojas, pero más angosta.

*Fundación R A. Philippi de Estudios Naturales. Apoquindo 6275 Of. 134. fundacionphilippi@infocon.cl

\begin{abstract}
Tecophilaea cyanocrocus Leyb. is an endemic geophyte to the high Andes in the Metropolitan Region of Chile. It was described in 1862. Due to the ornamental potential of its blue flowers, its corms were collected in large numbers and exported to Europe. Today it is cultivated in many countries. However, since mid 20th century, it has been considered extinct in the wild. In this publication, the finding in the spring of 2001 of a population of this species in the mountains of the Metropolitan Region of Chile is described.
\end{abstract}

Keywords: Flora of Chile, geophytes, extinct species, Tecophilaea cyanocrocus Leyb.

Son gamopétalas, infundibuliformes, poseen un tubo muy corto, 6 tépalos obovados de color azul intenso de $3 \mathrm{~cm}$ de largo por $1 \mathrm{~cm}$ en su parte más ancha, terminados en un mucrón. El androceo está formado por 3 estambres fértiles con anteras curvas caudadas y 3 estériles lanceoladas. El ovario es ínfero, 3 locular y pluriseminado, y el estilo es recto con estigma trífido. El fruto es una cápsula alargada coronada por la base del estilo, semillas negras.

Esta planta fue descrita por el farmacéutico y botánico alemán Friedrich Leybold en 1862, sobre la base de un ejemplar colectado en la cordillera de Santiago (Leybold 1862)

La familia Tecophilaceae Leyb. está representada en Chile por tres géneros endémicos: Conanthera, Tecophilaea y Zephyra. Todas ellas corresponden a geófitas de desarrollo primaveral en zonas árida, semiárida y mediterránea. Otros 
Gayana Bot. 59(2), 2002
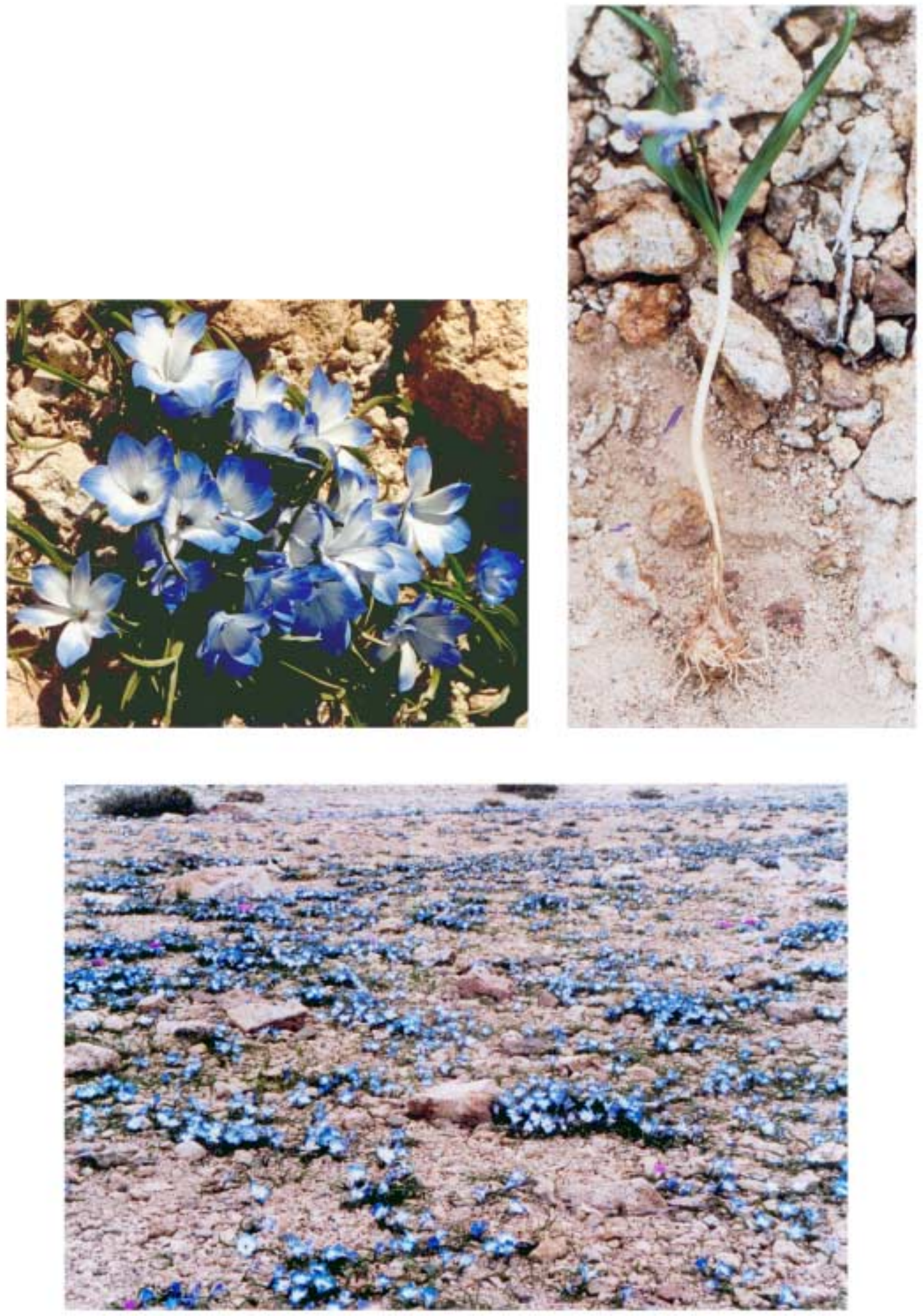

Figs. 1-3: Fig. 1. Tecophilaea cyanocrocus Leyb. (Fotografía R. García de la Huerta); Fig. 2. T. cyanocrocus Leyb. Planta completa (Fotografía M. T. Eyzaguirre); Fig. 3. Población de T. cyanocrocus Leyb. (Fotografía M. T. Eyzaguirre). 
géneros de esta familia están presentes en Africa y California (Mabberley 1998).

\section{ANTECEDENTES}

Desde su descubrimiento se hizo notar el valor comercial de sus flores. Ya en 1869 llegó una gran cantidad de cormos a Europa, al parecer importados por Max Leichtlin de Baden Baden, Alemania. Se trataba de 2.000 ejemplares que Leichtlin entregó a grandes firmas comerciales. Estos correspondían a T. cyanocrocus de tépalos azul con blanco, di-ferente al tipo enteramente azul, que para dis-tinguirlos denominó variedad leichtlinii. (Leichtlin 1889). En 1872 apareció dibujada en la revista Gartenflora (Regel 1872). Pocos años después se la presentó en las exposiciones de Londres.

La intensa colecta de los cormos y el pastoreo de ganado doméstico hizo que $T$. cyanocrocus desapareciera de su lugar de origen. Desde mediados del siglo XX en adelante se la considera extinguida en su hábitat natural (Muñoz 1973; Benoit 1989; Hoffmann 1989; Grau 1992; Hoffmann et al. 1998).

Crecía en una zona muy restringida en los alrededores de Santiago (Muñoz 1998); sin embargo, el lugar exacto no está determinado con claridad. Los cultivadores europeos (Leichtlin 1889; Reuthe 1889) la remiten a la Cordillera de Santiago y la Isla de Juan Fernández. Buscando aclarar este punto, se consultaron los herbarios chilenos SGO, CONC, VALD y SQF. Sólo se encontraron dos muestras de T. cyanocrocus en el primero de ellos. Una colectada en 1862 en la cordillera de Santiago y otra de 1878 proveniente de la hacienda Chicureo, la cual, a fines del siglo XIX, llegaba hasta la alta cordillera. En los herbarios europeos B y P existen también ejemplares, enviados por Philippi, sin fecha, provenientes de la Cordillera de Santiago, Chile, pero no existen más detalles sobre su origen. Aparentemente, la mención del Archipiélago de Juan Fernández tuvo por objeto confundir a la com-petencia comercial (P. F. Ravenna conv. pers.)

Actualmente es una especie muy apreciada por los horticultores de todo el mundo, recibiendo el nombre de "Chilean Blue Crocus". Se la considera una especie rara. En cultivo se distinguen tres variedades: la variedad tipo de flores azules, var. leichtlinii con perigonio azul y blanco y var. violacea de una tonalidad más violeta. Estos taxa no han sido publicados, correspondiendo sólo a denominaciones comerciales. Se le considera fácil de cultivar y se desarrolla bien en maceta, en una ubicación soleada. El sustrato debe permitir un buen drenaje y debe suspenderse el riego durante el letargo veraniego, además es necesario que pase un período de frío. Es resistente a plagas y enfermedades, salvo a la botritis. La floración es más abundante cuando recibe fertilizantes que incluyan $\mathrm{K}, \mathrm{P}$ y $\mathrm{Mg}$. Los cormos se multiplican muy lentamente siendo su reproducción más rápida por semilla, las cuales tienen una alta capacidad germinativa en un sustrato levemente ácido. Los nuevos ejemplares demoran 3 a 4 años en florecer. En el mercado internacional de plantas bulbosas hay una amplia oferta de cormos y semillas de las tres variedades antes mencionadas.

Existen iniciativas para reintroducir $T$. cyanocrocus a su hábitat natural. El Kew Garden de Londres, a través de Conservation Projects Developments Unit (CPDU), con la colaboración de la Corporación Nacional Forestal de Chile a través del Jardín Botánico de Viña del Mar y Alpine Garden Society, están formulando un proyecto para reintroducir in situ la especie. Sin embargo, hasta ahora no se tiene información de que se encuentre en cultivo en nuestro país.

\section{REDESCUBRIMIENTO}

La Fundación R. A. Philippi de Estudios Naturales realizó dos expediciones a un sector montañoso de la Región Metropolitana, ubicado a unos $40 \mathrm{~km}$ al sur de Santiago, durante la primavera de 2001. Durante ellas, pudo comprobar la existencia de una población de Tecophilaea cyanocrocus en estado silvestre. Se colectaron dos muestras; una fue depositada en SGO y otra en el Herbario de la Fundación.

El sector corresponde a una planicie cordillerana con afloramientos rocosos. Tiene una pendiente menor del $10 \%$ y está ubicada a 2.040 metros ms.n.m. Según la clasificación de capacidad de uso de suelo, éste corresponde al tipo VIII, sin valor agrícola, ganadero ni forestal (CIREN 1996).

De acuerdo a la clasificación de Köppen, el clima corresponde al tipo Csb1, templado cálido con una estación seca prolongada que coincide con el verano (Fuenzalida 1965). La temperatura media anual es de $14,5^{\circ} \mathrm{C}$. La precipitación media anual 
alcanza los 750 a $900 \mathrm{~mm}$, parte de ella en forma de nieve (CIREN 1996).

La región vegetacional corresponde a la Estepa Alto-Andina de la Cordillera de Santiago (Gajardo 1994). Presenta escasa cobertura vegetal. Las especies predominantes son Tetraglochim alatum y Baccharis sp., acompañantes son: Chuquiraga oppositifolia y Anarthrophyllum sp., todos arbustos con hábito de crecimiento en cojín. Escasa presencia de Laretia acaulis y Calandrinia cfr. affinis.

La población de $T$. cyanocrocus ocupa un sector claramente delimitado, de 20 por 50 metros. La cobertura es densa, existiendo entre 30 y 50 plantas por metro cuadrado, agrupadas de 5 a 20 cormos. La mayor parte de la planta está bajo tierra, sobresaliendo del suelo sólo $5 \mathrm{~cm}$. La parte subterránea es de longitud variable, influida por la presencia de piedras en el suelo. Una población más pequeña, integrada principalmente por individuos aislados, se encontró en un lugar cercano, en una hendidura por donde corrió agua producto del deshielo. En esta última, se asocia a Calandrinia cfr. affinis y Barneoudia major.

La morfología de la especie encontrada corresponde a T. cyanocrocus Leyb.; sin embargo hay diferencias en cuanto al color de los tépalos. El tubo es azul, los tépalos en su mayor parte blancos con las venas y el ápice azul intenso, lo cual coincide con la var. leichtlinii existente en cultivo. Este taxa infraespecífico no aparece en el Catálogo de la Flora Vascular de Chile (Marticorena y Quezada 1985), ya que nunca fue publicado. En la población más pequeña se encontraron 3 individuos con flores completamente blancas.

\section{CONCLUSIONES}

Tecophilaea cyanocrocus Leyb., considerada extinta durante más de 50 años, aún crece en forma silvestre en la naturaleza chilena. Este descubrimiento es relevante tratándose de una especie endémica que se creía perdida. Su estado de conservación pasa de la categoría extinta a la de rara y en grave peligro, dado lo escaso de sus poblaciones, por lo cuál, resulta urgente otorgarle la protección adecuada. El lugar donde se desarrolla es de propiedad privada y no tiene aptitud agropecuaria, sino sólo valor para la vida silvestre. Se reconoce una insuficiencia de áreas silvestres protegidas en la Región Metropolitana que representen los diferentes ecosistemas, e incluyan especies con serios peligros de conservación (CIREN 1994).

El sitio del hallazgo está muy alejado del lugar donde se la colectó en el siglo XIX, lo que extiende el rango de la especie hacia el sur.

La Fundación R. A. Philippi de Estudios Naturales considera importante dar a conocer este hallazgo al mundo científico. Sin embargo, se reserva los datos exactos de su ubicación, debido a que la especie no cuenta con la adecuada protección frente a su extrema vulnerabilidad.

\section{BIBLIOGRAFIA}

Benoit, I. L. (ED.). 1989. Libro Rojo de la Flora Terrestre de Chile (Primera parte). Santiago. 157 pp., 22 lám. incl. en el texto, 1 mapa.

Cheers, G. 1999. Botanica. The illustrated A - Z of over 10,000 garden plants and how to cultivated them. Welcome Rain, New York. 1,020 pp.

Ciren, 1994. Atlas forestal de Chile. Publicación CIREN-INFOR No 103.146 pp.

Ciren, 1996. Estudio Agrológico. Región Metropolitana. Descripciones de suelos. Materiales y Símbolos. 414 pp.

Fuenzalida, H. 1965. Clima. Geografía económica de Chile. CORFO. Santiago. Pp. 99-152.

Gajardo, R. 1994. La vegetación natural de Chile. Clasificación y distribución geográfica. Santiago. $165 \mathrm{pp}$.

Grau, J. 1992. Las monocotiledóneas petaloídeas de Chile. En J. Grau y G. Zizka (eds.) 1992. Flora silvestre de Chile. Palmengarten Sonderheft 19: 97-101.

Hoffmann, A. 1989. Chilean monocotyledonous geophytes. Taxonomic considerations and their state of conservation. Herbertia 45: 13-28.

Hoffmann, A.J., Liberona, F., \& Hoffmann, A.E. 1998. Distribution and Ecology of Geophytes in Chile. Conservation Threats to Geophytes in Mediterranean-Type Regions. Ecological Studies, Vol. 136: 231-253.

Leichtlin, M. 1889. Kleinere Mitteilungen. Gartenflora $38: 418,532$.

Leybold, F. 1862. Tecophilaea cyanocrocus Leyb. Bonplandia 10: 370.

Mabberley, D.J. 1998. The Plant-Book. A portable dictionary of the vascular plants. $2^{\circ}$ ed. Cambridge. xvi, $858 \mathrm{pp}$.

Marticorena, C. \& Quezada, M. 1985. Catálogo de la flora vascular de Chile. Gayana Botánica 42: 1-157.

MuÑoz, C.1973. Chile: plantas en extinción. Santiago. 248 pp., Editorial Universitaria, Santiago, Chile. 
Tecophilaea cyanocrocus redescubierta: EyzaguirRe, M. T. \& R. GARCíA dE LA Huerta

MuÑoz, M.1998. Plantas ornamentales nativas. Serie Forestal 10. Productos forestales no madereros en Chile. Dirección de productos forestales. FO, Roma. Oficina Regional de la FAO para América Latina y el Caribe. Santiago, Chile. 65 pp.

Regel, E. A. Von. 1872. Tecophilaea cyanocrocus Leyb. Gartenflora 21 : 97-98, t. 718.

Reuthe, G. 1889. Neue und empfehlenswerte Pflanzen. Gartenflora 38: 358-467.

\section{SITIOS WEB}

DıJK, B. 1998. Rare and Unusual Bulbs. Tecophilaea Species. NZ Garden Online. http://www.gardens.co.nz/ features/Tecophilaea.htm
Hardman,C. Living Jewels: Number 1 in a Series.

Tecophilaea cyanocrocus. Baldwin park, California, USA.http://www10.secure-website.net/ bulbsoc/ About_Bulbs/LIVING_JEWELS/TECOPHILAEA.htmL MuÑoz, M. \& MoreIRA, A. 2000. Los Géneros Endémicos de Monocotiledóneas de Chile Continental. http:// www.mnhn.cl

Royal Botanic Gardens. Royal Botanic Gardens Kew. Species Programme. http://www.rbgkew.org.uk/conservation/cpdu/ssp.html\#Tecophilea

Royal Botanic Gardens. Royal Botanic Gardens Kew. Conservation. http://www.rbgkew.org.uk/conservation/ cpdu/cpdu.html

THIMBLE FARMS 2001. The rare plant specialist. $2001 \mathrm{Bulb}$ list. http://www.thimblefarms.com/sbulb5.html

Fecha de recepción: 24.07.02

Fecha de aceptación: 04.09.02

Fecha de publicación: Abril de 2003. 